\title{
THE LAW OF EXPONENTIAL DECAY FOR EXPANDING TRANSFORMATIONS OF THE UNIT INTERVAL INTO ITSELF
}

BY

M. JABLOŃSKI

ABSTRACT. Let $T:[0,1] \rightarrow[0,1]$ be an expanding map of the unit interval and let $\xi_{\varepsilon}(x)$ be the smallest integer $n$ for which $T^{n}(x) \in[0, \varepsilon]$; that is, it is the random variable given by the formula

$$
\xi_{\varepsilon}(x)=\min \left\langle n: T^{n}(x) \leqslant \varepsilon\right\} .
$$

It is shown that for any $z \geqslant 0$ and for any integrable function $f:[0,1] \rightarrow R^{+}$the measure $\mu_{f}$ (where $\mu$ is Lebesgue measure and $\mu_{f}$ is defined by $d \mu_{f}=f d \mu$ ) of the set of points $x$ for which $\xi_{\varepsilon}(x) \leqslant z / \varepsilon$ tends to an exponential function of $z$ as $\varepsilon$ tends to zero.

1. Introduction. Denote by $\mu$ Lebesgue measure and by $L^{1}$ the space of all integrable functions defined on $[0,1]$. Let $f:[0,1] \rightarrow R^{+}$be an integrable function with $\|f\|=\int_{0}^{1} f d \mu=1$. Denote by $\mu_{f}$ the measure with density $f$; that is,

$$
\mu_{f}(A)=\int_{A} f d \mu
$$

for all measurable sets $A$.

In [1] A. Lasota and J. A. Yorke have proved that for any expanding transformation $T:[0,1] \rightarrow[0,1]$ there exists a constant $\sigma$ such that for any smooth function $f:$ $[0,1] \rightarrow R^{+}$such that $\int_{0}^{1} f d \mu=1$ and for any $z \geqslant 0$

$$
\lim _{\varepsilon \rightarrow 0} F_{\varepsilon}(f, z / \varepsilon)=1-e^{-\sigma z},
$$

where $F_{\varepsilon}(f, t)=\mu_{f}\left\{x: \xi_{\varepsilon}(x) \leqslant t\right\}, \xi_{\varepsilon}(x)=\max \left\{n: T^{k}(x) \leqslant 1\right.$ for all $\left.k=0,1, \ldots, n\right\}$ and $T_{\varepsilon}=(1+\varepsilon) T$. Using some generalizations of this theorem it is possible to explain some interesting physical and biological phenomena (see [10, 17]). The Lasota-Yorke Theorem is related to the family of transformations $T_{\varepsilon}$. It is quite natural to ask if the same kind of behavior, exponential decay, may be observed in dynamical systems governed by one transformation. The aim of the present paper is to give a partial positive answer to this question. It is interesting that this new problem requires also a new and more complicated technique. In our theorem we put $T_{\varepsilon} \equiv T$ instead of $T_{\varepsilon}=(1+\varepsilon) T$ and we define $\xi_{\varepsilon}$ by the following formula:

$$
\xi_{\varepsilon}(x)=\min \left\{n: T^{n}(x) \leqslant \varepsilon\right\} .
$$

Received by the editors May 28, 1982

1980 Mathematics Subject Classification. Primary 28D99. 
To prove this theorem we show that the function $F(z)=\lim _{\varepsilon \rightarrow 0} F_{\varepsilon}(f, z / \varepsilon)$ is a solution of the linear differential equation $F^{\prime}(z)=\sigma-\sigma F(z)$.

In $\$ 2$ we state the main theorem. In $\$ 3$ we prove some necessary lemmas and the theorem. In the last section we state final remarks.

2. The law of exponential decay for expanding transformations. Let $0=a_{0}<a_{1}<$ $\cdots<a_{m}=1$ be a partition of the unit interval and let $T:[0,1) \rightarrow[0,1)$ satisfy the following conditions:

(i) For any $i(i=1, \ldots, m)$ the restriction $T_{i}$ of $T$ to the interval $\left[a_{i-1}, a_{i}\right)$ is a bijection of class $C^{2}$ which can be extended to a function of class $C^{2}$ on the closed interval $\left[a_{i-1}, a_{i}\right]$.

(ii) $T_{i}\left(a_{i-1}\right)=0, T_{i}\left(\left[a_{i-1}, a_{i}\right)\right)=[0,1), i=1,2, \ldots, m$.

(iii) $s_{0}=\inf \left(T^{\prime}\right)>1$.

It is well known that for such a transformation $T$ there exists an absolutely continuous measure $\nu$ invariant under $T$ (i.e., $\nu\left(T^{-1}(A)\right)=\nu(A)$ for each measurable $A)$ with density $g_{\nu}($ see $[3,4])$ satisfying

$$
1 / c \leqslant g_{\nu} \leqslant c
$$

for some $c>0$. Moreover, $g_{\nu}$ is continuous (see [8]).

Put $\sigma=g_{\nu}(0)\left(1-\left(T_{1}^{-1}\right)^{\prime}(0)\right)$. We have the following

THEOREM 1. If T: $[0,1) \rightarrow[0,1)$ satisfies condition (i)-(iii), then, for any nonnegative $f \in L^{1}$ such that $\|f\|=1$ and for any $z \geqslant 0$, we have

$$
\lim _{\varepsilon \rightarrow 0} F_{\varepsilon}(f, z / \varepsilon)=1-e^{-\sigma z},
$$

where $F_{\varepsilon}(f, t)=\mu_{f}\left\{x: \xi_{\varepsilon}(x) \leqslant t\right\}$.

REMARK. It is easy to see that

$$
F_{\varepsilon}(f, t)=\mu_{f} \bigcup_{0 \leqslant n \leqslant t} T^{-n}([0, \varepsilon]) .
$$

Therefore, the thesis of Theorem 1 may also be expressed by the formula

$$
\lim _{\varepsilon \rightarrow 0} \mu_{f}\left(\bigcup_{0 \leqslant n \leqslant z / \varepsilon} T^{-n}([0, \varepsilon])\right)=1-e^{-\sigma z} .
$$

3. Auxiliary lemmas and proof of Theorem 1. Let $T:[0,1) \rightarrow[0,1)$ be a measurable nonsingular function, i.e., if $A$ is measurable, $\mu(A)=0$ implies $\mu\left(T^{-1}(A)\right)=0$. Given $T$, the Frobenius-Perron operator $P_{T}: L^{1} \rightarrow L^{1}$ is given by the formula

$$
\left(P_{T} f\right)(x)=\frac{d}{d x} \int_{T^{-1}([0, x))} f(s) d s .
$$

The operator $P_{T}$ is linear, continuous and satisifes the following conditions:

(a) $P_{T}$ is positive: $f \geqslant 0 \Rightarrow P_{T} f \geqslant 0$.

(b) $P_{T}$ preserves the integral

$$
\int_{0}^{1} P_{T} f d \mu=\int_{0}^{1} f d \mu, \quad f \in L^{1} .
$$


(c) $P_{T^{n}}=P_{T}^{n}$, where $T^{n}$ denotes the $n$th iterate of $T$.

(d) $P_{T} f=f$ iff the measure $d \nu_{T}=f d \mu$ is invariant under $T$.

(e) For any $f \in L^{1}$ and for any $g \in L^{\infty}, \int_{0}^{1} g \cdot P_{T} f d \mu=\int_{0}^{1}(g \circ T) f d \mu$.

We also have the following

THEOREM 2. If $T$ satisfies conditions (i)-(iii), then there exist constants $K>0$, $C>0$ and $0<s<1$ such that for any nonnegative function $f$ with bounded variation on $[0,1]$

$$
\left|P_{T}^{n} f-g_{\nu}\|f\|\right| \leqslant s^{n} K(\underset{0}{\vee} f+C\|f\|),
$$

where $\vee_{0}^{1}$ denotes the variation of $f$ over $[0,1]$.

The proof of this theorem is given in [5].

Let $T$ satisfy conditions (i)-(iii) and let $Q_{n}$ denote the $n$th Cartesian product of the set $\{1,2, \ldots, m\}$. For any $i \in Q_{1}=\{1,2, \ldots, m\}$ and any $\left(i_{1}, i_{2}, \ldots, i_{r}\right) \in Q_{r}$ put $\varphi_{i}=T_{i}^{-1}, \varphi_{i_{1}, \ldots, i_{r}}=\varphi_{i_{r}} \circ \cdots \circ \varphi_{i_{1}}$ and $\Delta_{i_{1}, \ldots, i_{r}}=\varphi_{i_{1}, \ldots, i_{r}}([0,1))$. It is obvious that $\Delta_{i_{1}, \ldots, i_{r}}$ are intervals, their end points form the partition corresponding to the transformation $T^{r}$ and

$$
\left(\left.T^{r}\right|_{\Delta_{i_{1} \ldots, i_{r}}}\right)^{-1}=\varphi_{i_{1} \ldots, i_{r}}
$$

LEMMA 1. There exists a constant $M$ such that for every $r>0$ and every $\left(i_{1}, i_{2}, \ldots\right.$, $\left.i_{r}\right) \in Q_{r}$

$$
\underset{0}{\vee}\left(g_{\nu} \circ \varphi_{i_{1}, \ldots, i_{r}} \frac{\left|\varphi_{i_{1}, \ldots, i_{r}}^{\prime}\right|}{\nu\left(\Delta_{i_{1}, \ldots, i_{r}}\right)}\right) \leqslant M
$$

Proof. For some constant $L$ we have

$$
\left|\frac{\varphi_{i_{1}, \ldots, i_{r}}^{\prime}(x)}{\varphi_{i_{1}, \ldots, i_{r}}^{\prime}(y)}\right| \leqslant L
$$

whenever $x, y \in[0,1), r>0$ and $\left(i_{1}, \ldots, i_{r}\right) \in Q_{r}$ (see [7]). Since $\vee_{0}^{1} g_{\nu}<\infty$ (see [2]) and

$$
\underset{0}{\vee}\left(g_{\nu} \circ \varphi_{i_{1}, \ldots, i_{r}} \frac{\left|\varphi_{i_{1}, \ldots, i_{r}}^{\prime}\right|}{\nu\left(\Delta_{i_{1}, \ldots, i_{r}}\right)}\right) \leqslant \underset{0}{\vee} g_{\nu} \sup \frac{\left|\varphi_{i_{1}, \ldots, i_{r}}^{\prime}\right|}{\nu\left(\Delta_{i_{1}, \ldots, i_{r}}\right)}+\left(\sup g_{\nu}\right) \underset{0}{\stackrel{1}{\bigvee}} \frac{\left|\varphi_{i_{1}, \ldots, i_{r}}^{\prime}\right|}{\nu\left(\Delta_{i_{1}, \ldots, i_{r}}\right)},
$$

to prove the lemma it is sufficient to show that there exists a constant $M_{1}$ such that for every $r>0$ and every $\left(i_{1}, i_{2}, \ldots, i_{r}\right) \in Q_{r}$

$$
\sup _{x \in[0,1)} \frac{\left|\varphi_{i_{1}, \ldots, i_{r}}^{\prime}\right|}{\nu\left(\Delta_{i_{1}, \ldots, i_{r}}\right)} \leqslant M_{1}
$$

and

$$
\bigvee_{0}^{1} \frac{\left|\varphi_{i_{1}, \ldots, i_{r}}^{\prime}\right|}{\nu\left(\Delta_{i_{1}, \ldots, i_{r}}\right)} \leqslant M_{1}
$$


By the mean value theorem there exists $\theta \in(0,1)$ such that

$$
\mu\left(\Delta_{i_{1}, \ldots, i_{r}}\right)=\varphi_{i_{1}, \ldots, i_{r}}^{\prime}(\theta) .
$$

Thus, by (1) and (4) we obtain (5).

We have

$$
{\underset{0}{0}}_{0}^{1} \frac{\left|\varphi_{i_{1}, \ldots, i_{r}}^{\prime}\right|}{\nu\left(\Delta_{i_{1}, \ldots, i_{r}}\right)}=\frac{1}{\nu\left(\Delta_{i_{1}, \ldots, i_{r}}\right)} \int_{0}^{1}\left|\varphi_{i_{1}, \ldots, i_{r}}^{\prime \prime}\right| d s,
$$

and, moreover, by the assumptions on $T$

$$
\begin{aligned}
\left|\varphi_{i_{1}, \ldots, i_{r}}^{\prime \prime}\right| & =\left|\left(\prod_{j=1}^{r} \varphi_{i_{j}}^{\prime} \circ \varphi_{i_{1}, \ldots, i_{j-1}}\right)^{\prime}\right| \\
& =\mid \sum_{j=1}^{r}\left(\prod_{p=1}^{j-1} \varphi_{i_{p}}^{\prime} \circ \varphi_{i_{1} \ldots, i_{p-1}}\left|\left(\varphi_{i_{j}}^{\prime \prime} \circ \varphi_{i_{1}, \ldots, i_{j-1}}\right) \varphi_{i_{1}, \ldots, i_{j-1}}^{\prime}\left(\prod_{p=j+1}^{r} \varphi_{i_{p}}^{\prime} \circ \varphi_{i_{1}, \ldots, i_{p-1}}\right)\right|\right. \\
& =\left|\prod_{j=1}^{r} \varphi_{i_{j}}^{\prime} \circ \varphi_{i_{1}, \ldots, i_{j-1}} \sum_{j=1}^{r} \frac{\left(\varphi_{i_{j}}^{\prime \prime} \circ \varphi_{i_{1}, \ldots, i_{j-1}}\right) \varphi_{i_{1}, \ldots, i_{j-1}}^{\prime}}{\varphi_{i_{j}}^{\prime} \circ \varphi_{i_{1}, \ldots, i_{j-1}}}\right| \\
& \leqslant\left|\varphi_{i_{1}, \ldots, i_{r}}^{\prime}\right| \sum_{j=1}^{r}\left|\frac{\sup \varphi_{i_{j}}^{\prime \prime} \mid}{\inf \varphi_{i_{j}}^{\prime}}\right|\left|\varphi_{i_{1}, \ldots, i_{j-1}}^{\prime}\right| \\
& \leqslant\left|\varphi_{i_{1}, \ldots, i_{r}}^{\prime}\right| \sup _{j}\left[\left(\sup _{x \in[0,1)} \varphi_{j}^{\prime \prime}\right)\left(\inf _{x \in[0,1)} \varphi_{j}^{\prime}\right)^{-1}\right] \sum_{j=1}^{r} s_{0}^{-j} \\
& \leqslant\left|\varphi_{i_{1}, \ldots, i_{r}}^{\prime}\right| M_{2}
\end{aligned}
$$

for some $M_{2}$. Therefore, by (1), (4), (7) and (8) we have

$$
\begin{aligned}
{\underset{0}{\mathrm{~V}}}_{0}^{\mathrm{V}} \frac{\varphi_{i_{1}, \ldots, i_{r}}^{\prime}}{\nu\left(\Delta_{i_{1}, \ldots, i_{r}}\right)} & \leqslant c \mu\left(\Delta_{i_{1}, \ldots, i_{r}}\right) M_{2} \int_{0}^{1}\left|\varphi_{i_{1}, \ldots, i_{r}}^{\prime}\right| d s \\
& \leqslant c M_{2} \int_{0}^{1}\left|\frac{\varphi_{i_{1}, \ldots, i_{r}}^{\prime}}{\varphi_{i_{1}, \ldots, i_{r}}^{\prime}(\theta)}\right| d s \leqslant c M_{2} L .
\end{aligned}
$$

This ends the proof of the lemma.

For $T$ satisfying conditions (i)-(iii) we define $\eta\left(i_{1}, \ldots, i_{r}, q, \varepsilon\right)$ by

$$
\begin{aligned}
& \nu\left(\Delta_{i_{1}, \ldots, i_{r}} \cap T^{-q}([0, \varepsilon])\right) \\
& \quad=\nu\left(\Delta_{i_{1}, \ldots, i_{r}}\right) \nu([0, \varepsilon])+\nu\left(\Delta_{i_{1}, \ldots, i_{r}}\right) \nu([0, \varepsilon]) \eta\left(i_{1}, \ldots, i_{r}, q, \varepsilon\right) .
\end{aligned}
$$

It is well known that the transformation $T$ is mixing; that is,

$$
\lim _{n \rightarrow \infty} \nu\left(A \cap T^{-n}(B)\right)=\nu(A) \nu(B)
$$


whenever $A \subset[0,1)$ and $B \subset[0,1)$ are measurable. The following lemma gives us an estimation of the rate of convergence of the sequence $\nu\left(A \cap T^{-n}(B)\right)$ with $A=\Delta_{i_{1}, \ldots, i_{r}}$ and $B=[0, \varepsilon]$.

LEMma 2. There exists $K>0$ and $0<s<1$ such that for $q>r$,

$$
\max \left\{\left|\eta\left(i_{1}, \ldots, i_{r}, q, \varepsilon\right)\right|:\left(i_{1}, \ldots, i_{r}\right) \in Q_{r}\right\} K s^{q-r} .
$$

Proof. By simple computations we obtain

$$
P_{T}^{r} f=\sum_{i_{1}, \ldots, i_{r}=1}^{m} f\left(\varphi_{i_{1}, \ldots, i_{r}}\right)\left|\varphi_{i_{1}, \ldots, i_{r}}^{\prime}\right| .
$$

From (e) we have

$$
\begin{aligned}
\nu\left(\Delta_{i_{1}, \ldots, i_{r}} \cap T^{-q}([0, \varepsilon])\right) & =\int_{0}^{1} 1_{i_{1} \ldots, i_{r}} 1_{[0, \varepsilon]}\left(T^{q}\right) d \nu \\
& =\int_{0}^{1} P_{T}^{q}\left(g_{\nu} 1_{i_{1}, \ldots, i_{r}}\right) 1_{[0, \varepsilon]} d \mu,
\end{aligned}
$$

where $1_{i_{1}, \ldots, i_{r}}$ and $1_{[0, \varepsilon]}$ denote the characteristic functions of the intervals $\Delta_{i_{1}, \ldots, i_{r}}$ and $[0, \varepsilon]$, respectively. Therefore

$$
\begin{aligned}
\left|\nu\left(\Delta_{i_{1}, \ldots, i_{r}} \cap T^{-q}([0, \varepsilon])\right)-\nu\left(\Delta_{i_{1}, \ldots, i_{r}}\right) \nu([0, \varepsilon])\right| \\
\quad=\left|\int_{0}^{1} P_{T}^{q}\left(g_{\nu} 1_{i_{1} \ldots, i_{r}}\right) 1_{[0, \varepsilon]} d \mu-\int_{0}^{1} \nu\left(\Delta_{i_{1} \ldots, i_{r}}\right) 1_{[0, \varepsilon]} g_{\nu} d \mu\right| \\
\quad \leqslant \int_{0}^{1}\left|1_{[0, \varepsilon]}\left(P_{T}^{q}\left(g_{\nu} 1_{i_{1} \ldots, i_{r}}\right)-g_{\nu} \nu\left(\Delta_{i_{1} \ldots, i_{r}}\right)\right)\right| d \mu \\
\quad \leqslant \mu([0, \varepsilon]) \sup _{x \in[0,1)}\left|P_{T}^{q}\left(g_{\nu} 1_{i_{1}, \ldots, i_{r}}\right)-g_{\nu} \nu\left(\Delta_{i_{1} \ldots, i_{r}}\right)\right| .
\end{aligned}
$$

Moreover, by (9), the definition of $\varphi_{i_{1}, \ldots, i_{r}}$ and the definition of $\Delta_{i_{1} \ldots, i_{r}}$, we obtain

$$
P_{T}^{r}\left(\frac{g_{\nu} 1_{i_{1}, \ldots, i_{r}}}{\nu\left(\Delta_{i_{1}, \ldots, i_{r}}\right)}\right)=\frac{g_{\nu}\left(\varphi_{i_{1}, \ldots, i_{r}}\right)\left|\varphi_{i_{1}, \ldots, i_{r}}^{\prime}\right|}{\nu\left(\Delta_{i_{1} \ldots, i_{r}}\right)}
$$

Since

$$
\left|P q\left(g_{\nu} 1_{i_{1}, \ldots, i_{r}}\right)-g_{\nu} \nu\left(\Delta_{i_{1}, \ldots, i_{r}}\right)\right|=\nu\left(\Delta_{i_{1}, \ldots, i_{r}}\right)\left|P_{T}^{q-r}\left(P_{T}^{r}\left(\frac{g_{\nu} 1_{i_{1}, \ldots, i_{r}}}{\nu\left(\Delta_{i_{1}, \ldots, i_{r}}\right)}\right)\right)-g_{\nu}\right|
$$

and

$$
\int_{0}^{1} P_{T}^{r}\left(\frac{g_{\nu} 1_{i_{1} \ldots, i_{r}}}{\nu\left(\Delta_{i_{1} \ldots, i_{r}}\right)}\right) d \mu=\int_{0}^{1} \frac{g_{\nu} 1_{i_{1} \ldots, i_{r}}}{\nu\left(\Delta_{i_{1} \ldots, i_{r}}\right)} d \mu=1
$$


by (d), (11), Lemma 1 and Theorem 2 we have, for some $M>0$ and $s \in(0,1)$,

$$
\begin{aligned}
\sup \mid & P_{T}^{q}\left(g_{\nu} 1_{i_{1} \ldots, i_{r}}\right)-g_{\nu} \nu\left(\Delta_{i_{1} \ldots, i_{r}}\right) \mid \\
& =\nu\left(\Delta_{i_{1} \ldots, i_{r}}\right) \sup \left|P_{T}^{q-r}\left(P_{T}^{r}\left(\frac{g_{\nu} 1_{i_{1}, \ldots, i_{r}}}{\nu\left(\Delta_{i_{1}, \ldots, i_{r}}\right)}\right)-g_{\nu}\right)\right| \\
& \leqslant \nu\left(\Delta_{i_{1} \ldots, i_{r}}\right) s^{q-r}\left(\begin{array}{c}
1 \\
\vee
\end{array}\left(\frac{g_{\nu}\left(\varphi_{i_{1}, \ldots, i_{r}}\right) \mid \varphi_{i_{1}, \ldots, i_{r}}^{\prime}}{\nu\left(\Delta_{i_{1} \ldots, i_{r}}\right)}\right)+C\right) \\
& \leqslant \nu\left(\Delta_{i_{1}, \ldots, i_{r}}\right)(M+C) s^{q-r} .
\end{aligned}
$$

This, (1) and (10) give us the thesis of the lemma.

For the sake of convenience, set $\Delta_{i_{1}, \ldots, i_{r}}=\Delta_{w}$, where $w=\left(i_{1}, \ldots, i_{r}\right) \in Q_{r}$.

LEMMA 3. If T satisfies conditions (i)-(iii), then, for every $\varepsilon \in(0,1)$, the measure $\nu$ of the union of the intervals $\Delta_{w}$ such that

$$
\bigcup_{k=0}^{p} T^{-k}(\{\varepsilon\}) \cap \Delta_{w} \neq 0
$$

tends to zero geometrically as $r-p \rightarrow \infty$; that is, there exists a constant $M$ independent of $p, \varepsilon$ and $r$ such that, for $r>p$,

$$
\sum_{k=0}^{p} \sum_{\Delta_{w} \cap T^{-k}(\{\varepsilon\}) \neq 0} \nu\left(\Delta_{w}\right) \leqslant M s_{0}^{p-r} .
$$

Proof. It is obvious that $\Delta_{i_{1}, \ldots, i_{r}} \cap T^{-k}(\{\varepsilon\}) \neq 0$ iff $\varepsilon \in T^{k}\left(\Delta_{i_{1}, \ldots, i_{r}}\right)=\Delta_{i_{1}, \ldots, i_{r-k}}$ and, moreover, $T^{k}\left(\Delta_{i_{1}, \ldots, i_{r}}\right)=T^{k}\left(\Delta_{j_{1}, \ldots, j_{r}}\right)$ iff $i_{1}=j_{1}, \ldots, i_{r-k}=j_{r-k}$. Hence, since $\Delta_{i_{1}, \ldots, i_{r-k}}$ are disjoint sets, there exists $w_{0}=\left(i_{1}^{0}, \ldots, i_{r-k}^{0}\right) \in Q_{r-k}$ such that $\Delta_{i_{1}, \ldots, i_{r}}$ $\cap T^{-k}(\{\varepsilon\}) \neq \varnothing$ iff $i_{1}=i_{1}^{0}, \ldots, i_{r-k}=i_{r-k}^{0}$; that is, $\Delta_{i_{1}, \ldots, i_{r}} \cap T^{-k}(\{\varepsilon\}) \neq \varnothing$ iff $T^{k}\left(\Delta_{i_{1}, \ldots, i_{r}}\right)=\Delta_{w_{0}}$. Therefore

$$
\bigcup_{\Delta_{w} \cap T^{-k}(\{\varepsilon\}) \neq 0} \Delta_{w}=\bigcup_{i_{r-k+1}, \ldots, i_{r}=1}^{m} \varphi_{i_{r-k+1} \ldots, i_{r}}\left(\Delta_{w_{0}}\right)=T^{-k}\left(\Delta_{w_{0}}\right)
$$

and, consequently, since $T$ preserves $\nu, \mu\left(\Delta_{i_{1} \ldots, i_{n}}\right) \leqslant s_{0}^{-n}$ and $\Delta_{w}$ are disjoint sets, by (1) we have

$$
\sum_{\Delta_{w_{0}} \cap T^{-k}(\langle\varepsilon\}) \neq 0} \nu\left(\Delta_{w}\right)=\nu\left(T^{-k}\left(\Delta_{w_{0}}\right)\right)=\nu\left(\Delta_{w_{0}}\right) \leqslant c \mu\left(\Delta_{w_{0}}\right) \leqslant c s_{0}^{k-r} .
$$

This implies the thesis of the lemma.

Put $B(i, \varepsilon)=T^{-i}([0, \varepsilon])$ and $A(k, p, \varepsilon)=\cup_{i=k}^{p} B(i, \varepsilon)$. Let the function $R\left(p, q, \varepsilon_{2}\right)$ be given by the formula

$\nu\left(A\left(0, p, \varepsilon_{1}\right) \cap B\left(q, \varepsilon_{2}\right)\right)=\nu\left(A\left(0, p, \varepsilon_{1}\right)\right) \nu\left(\left[0, \varepsilon_{2}\right]\right)+R\left(p, q, \varepsilon_{2}\right) \nu\left(\left[0, \varepsilon_{2}\right]\right)$.

An estimation similar to that of Lemma 2 is given in the following. 
LEMMA 4. For $T$ satisfying conditions (i)-(iii) there exist constants $M$ and $0<s<1$ such that, for $p<q$ and $\varepsilon_{2} \in(0,1)$, we have

$$
\left|R\left(p, q, \varepsilon_{2}\right)\right| \leqslant M s^{(q-p) / 2} .
$$

Proof. Let $r$ be a natural number such that $p<r<q$ and let $E_{1}$ be the union of the intervals $\Delta_{i_{1} \ldots, i_{r}}$ such that $\Delta_{i_{1} \ldots, i_{r}} \subset A\left(0, p, \varepsilon_{1}\right)$; that is,

$$
E_{1}=\bigcup_{\Delta_{i_{1} \ldots, i_{r}} \subset A\left(0, p, \varepsilon_{1}\right)} \Delta_{i_{1} \ldots, i_{r}}
$$

We denote by $E_{2}$ the union of the intervals $\Delta_{i_{1}, \ldots, i_{r}}$ such that $\Delta_{i_{1}, \ldots, i_{r}} \cap A\left(0, p, \varepsilon_{1}\right) \neq$ $\varnothing$ and $\Delta_{i_{1}, \ldots, i_{r}} \not \subset A\left(0, p, \varepsilon_{1}\right)$. It is obvious that, for every interval $\Delta_{i_{1}, \ldots, i_{r}}$ from the union $E_{2}$, there exists $n \leqslant p$ such that $\Delta_{i_{1}, \ldots, i_{r}} \cap T^{-n}\left(\left\{\varepsilon_{1}\right\}\right) \neq \varnothing$ and, moreover, by Lemma 3

$$
\nu\left(E_{2}\right) \leqslant M s_{0}^{p-r}
$$

for some $M$ independent of $p, r$ and $\varepsilon_{1}$. Since $E_{1} \cap E_{2}=0$, for $w \in Q_{r}$, we have

$$
\begin{aligned}
\left|\nu\left(A\left(0, p, \varepsilon_{1}\right) \cap B\left(q, \varepsilon_{2}\right)\right)-\nu\left(A\left(0, p, \varepsilon_{1}\right)\right) \nu\left(\left[0, \varepsilon_{2}\right]\right)\right| & -\nu\left(E_{2} \cap A\left(0, p, \varepsilon_{1}\right)\right) \nu\left(\left[0, \varepsilon_{2}\right]\right) \mid \\
= & \mid \nu\left(E_{1} \cap B\left(q, \varepsilon_{2}\right)\right)+\nu\left(E_{2} \cap A\left(0, p, \varepsilon_{1}\right) \cap B\left(q, \varepsilon_{2}\right)\right)-\nu\left(E_{1}\right) \nu\left(\left[0, \varepsilon_{2}\right]\right) \\
= & \mid \sum_{\Delta_{w} \subset E_{1}} \nu\left(\Delta_{w} \cap B\left(q, \varepsilon_{2}\right)\right)+\sum_{\Delta_{w} \subset E_{2}} \nu\left(\Delta_{w} \cap A\left(0, p, \varepsilon_{1}\right) \cap B\left(q, \varepsilon_{2}\right)\right)
\end{aligned}
$$

Hence, putting $r=\max \{n \leqslant(q+p) / 2\}$, by Lemma 2 and (12) we obtain the thesis of the lemma.

It is obvious that, for $T$ satisfying conditions (i)-(iii), the sequence $\varphi_{1}^{n}\left(a_{1}\right)$ is decreasing and $\varphi_{1}^{n}\left(a_{1}\right) \rightarrow 0$ as $n \rightarrow \infty$. For $\varepsilon<a_{1}$ put

$$
u(\varepsilon)=\max \left\{n \in N: \varphi_{1}^{n}\left(a_{1}\right)>\varepsilon\right\} .
$$


LEMMA 5. For any $p>u(\varepsilon)+1$ we have

$$
\begin{aligned}
\nu(A(0, p+1, \varepsilon))-\nu(A(0, p, \varepsilon)) \\
=\left[\nu([0, \varepsilon])-\nu\left(\varphi_{1}([0, \varepsilon])\right)\right][1-\nu(A(0, p-u(\varepsilon)+1, \varepsilon))] \\
\quad+R\left(p-u(\varepsilon)+1, p, \varphi_{1}(\varepsilon)\right) \nu\left(\left[0, \varphi_{1}(\varepsilon)\right]\right) \\
\quad-R(p-u(\varepsilon)+1, p+1, \varepsilon) \nu([0, \varepsilon]) .
\end{aligned}
$$

REMARK. If we divide the last equality by $\varepsilon$ we obtain an approximation of a differential equation for which the limit function $F\left(g_{\nu}, z\right)=\lim _{\varepsilon \rightarrow 0} F_{\varepsilon}\left(g_{\nu}, z / \varepsilon\right)$ is a solution.

Proof of Lemma 5. Let $\varepsilon<a_{1}$. It is obvious that

$$
\nu\left(T^{-1}([0, \varepsilon]) \backslash[0, \varepsilon]\right)=\nu([0, \varepsilon])-\nu\left(\varphi_{1}([0, \varepsilon])\right) .
$$

Therefore, since $T$ preserves $\nu$,

$$
\begin{aligned}
\nu\left(T^{-i-1}([0, \varepsilon]) \backslash T^{-i}([0, \varepsilon])\right) & =\nu\left(T^{-i}\left(T^{-1}([0, \varepsilon]) \backslash[0, \varepsilon]\right)\right) \\
& =\nu([0, \varepsilon])-\nu\left(\varphi_{1}([0, \varepsilon])\right) .
\end{aligned}
$$

By the definition of $u(\varepsilon)$, for $1 \leqslant i<u(\varepsilon)$, we have

$$
\left(T^{-i}([0, \varepsilon]) \backslash T^{-i+1}([0, \varepsilon])\right) \cap[0, \varepsilon]=\varnothing
$$

and, consequently,

$$
\left(T^{-p-1}([0, \varepsilon]) \cap T^{-p}([0, \varepsilon])\right) \cap T^{-j}([0, \varepsilon])=\varnothing
$$

whenever $p-j<u(\varepsilon)-1$. Equalities (13) and (14) for $p-k<u(\varepsilon)-1$ give us

$$
\nu(A(k, p+1, \varepsilon) \backslash A(k, p, \varepsilon))=\nu([0, \varepsilon])-\nu\left(\varphi_{1}([0, \varepsilon])\right) .
$$

It is easy to see that, for $i<u(\varepsilon)$,

$$
[0, \varepsilon] \cap T^{-i}([0, \varepsilon])=\varphi_{1}^{i}([0, \varepsilon]) .
$$

Therefore, for $p-i<u(\varepsilon)-1$, we have

$$
\begin{aligned}
T^{-i}([0, \varepsilon]) \cap T^{-p-1}([0, \varepsilon]) & =T^{-i}([0, \varepsilon]) \cap T^{-p-1+i}([0, \varepsilon]) \\
& =T^{-i}\left(\varphi^{p+1-i}([0, \varepsilon])\right) .
\end{aligned}
$$

Moreover, since

$$
T^{-j}\left(\varphi_{1}^{p+1-j}([0, \varepsilon])\right)=\bigcup_{i_{1}, \ldots, i_{j}=1}^{m} \varphi_{i_{1}, \ldots, i_{j}}\left(\varphi_{1}^{p+1-j}([0, \varepsilon])\right)
$$

we have, for $i>j$,

$$
T^{-j}\left(\varphi P^{+1-j}([0, \varepsilon])\right) \subset T^{-i}\left(\varphi P^{+1-i}([0, \varepsilon])\right) .
$$

From (16) and (17), for $p-k<u(\varepsilon)-1$, we obtain

$$
A(k, p, \varepsilon) \cap T^{-p-1}([0, \varepsilon])=T^{-p}\left(\varphi_{1}([0, \varepsilon])\right) .
$$

It is easy to verify that

$$
\nu(A \cup B \cup C)-\nu(A \cup B)=\nu((B \cup C) \backslash B)-\nu(A \cap C)+\nu(A \cap B \cap C) \text {. }
$$


Thus, setting $A=A(0, p-u(\varepsilon)+1, \varepsilon), B=A(p-u(\varepsilon)+2, p, \varepsilon)$ and $C=$ $T^{-p-1}([0, \varepsilon])$, from (15), (18) and (19) we have

$$
\begin{aligned}
& \nu(A(0, p+1, \varepsilon))-(A(0, p, \varepsilon)) \\
&= \nu(A(p-u(\varepsilon)+2, p+1, \varepsilon) \backslash A(p-u(\varepsilon)+2, p, \varepsilon)) \\
&-\nu\left(A(0, p-u(\varepsilon)+1, \varepsilon) \cap T^{-p-1}([0, \varepsilon])\right) \\
&+\nu\left(A(0, p-u(\varepsilon)+1, \varepsilon) \cap A(p-u(\varepsilon)+2, p, \varepsilon) \cap T^{-p-1}([0, \varepsilon])\right) \\
&= \nu([0, \varepsilon])-\nu\left(\varphi_{1}([0, \varepsilon])\right)-\nu(A(0, p-u(\varepsilon)+1, \varepsilon)) \nu([0, \varepsilon]) \\
&-R(p-u(\varepsilon)+1, p+1, \varepsilon) \nu([0, \varepsilon]) \\
&+\nu(A(0, p-u(\varepsilon)+1, \varepsilon)) \nu\left(\varphi_{1}([0, \varepsilon])\right) \\
&+R\left(p-u(\varepsilon)+1, p, \varphi_{1}(\varepsilon)\right) \nu\left(\left[0, \varphi_{1}(\varepsilon)\right]\right) .
\end{aligned}
$$

This gives us the thesis of the lemma.

A sequence of functions $\left\{f_{n}\right\}_{n=1}^{\infty}, f_{n}:[a, b] \rightarrow R$, is said to be quasi-equicontinuous on $[a, b]$ if for every $\eta>0$ there exist $n_{0}$ and $\delta>0$ such that

$$
\left|f_{n}(x)-f_{n}(y)\right|<\eta
$$

whenever $|x-y|<\delta, x, y \in[a, b]$, and $n>n_{0}$.

We shall need the following generalization of the Arzela Theorem.

ThEOREM (ARZELA). If a sequence $\left\{f_{n}\right\}_{n=1}^{\infty}, f_{n}:[a, b] \rightarrow R$, is uniformly bounded on $[a, b]$ and quasi-continuous on $[a, b]$, then:

(i) $\left\{f_{n}\right\}_{n=1}^{\infty}$ contains a uniformly convergent subsequence $\left\{f_{n_{j}}\right\}$.

(ii) $\lim _{j \rightarrow \infty} f_{n_{j}}$ is a continuous function.

The proof of this theorem is identical with that of the well-known Arzela Theorem.

The following two lemmas are easy to verify.

LEMMA 6. Let $\left\{f_{n}\right\}_{n=1}^{\infty}$ be a sequence of piecewise linear and continuous functions from $[a, b]$ into $R$. If $f_{n}$ is uniformly convergent to a function $f$ and the sequences $\left\{f_{n+}^{\prime}\right\}_{n=1}^{\infty}$ and $\left\{f_{n-}^{\prime}\right\}_{n=1}^{\infty}$ of the right and the left derivatives of the functions $f_{n}$ are uniformly convergent to a continuous function $\bar{f}$, then $f$ has the continuous derivative and

$$
\bar{f}=\lim _{n \rightarrow \infty} f_{n+}^{\prime}=\lim _{n \rightarrow \infty} f_{n-}^{\prime}=f^{\prime} .
$$

LEMMA 7. Let us consider a family of functions $\left\{f_{\varepsilon}\right\}_{\varepsilon \in(0,1]}$ such that $f_{\varepsilon}:[0, \infty) \rightarrow[0,1]$. If there exists a function $f$ such that for any sequence $\varepsilon_{n} \rightarrow 0$ and any $a>0$ there exists a subsequence $\varepsilon_{n_{j}}$ of $\varepsilon_{n}$ such that $f_{\varepsilon_{n_{j}}} \rightarrow f$ uniformly on $[0, a]$, then there exists $\lim _{\varepsilon \rightarrow 0} f_{\varepsilon}$ and, for any $a>0, f_{\varepsilon}$ converges uniformly on $[0, a]$.

Now, we prove Theorem 1 in the case where $f=g_{\nu}$.

LEMMA 8. If $T$ satisfies conditions (i)-(iii), then for $z \geqslant 0$

$$
\lim _{\varepsilon \rightarrow 0} F_{\varepsilon}\left(g_{\nu}, z / \varepsilon\right)=1-e^{-\sigma z},
$$

where $\sigma=g_{\nu}(0)\left(1-\varphi_{1}^{\prime}(0)\right)$. 
Proof. Put $F_{\varepsilon}(z / \varepsilon)=F_{\varepsilon}\left(g_{\nu}, z / \varepsilon\right)$. According to the Remark which follows the statement of Theorem $1, F_{\varepsilon}(z / \varepsilon)$ is given by the formula

$$
F_{\varepsilon}(z / \varepsilon)=\nu\left(\bigcup_{0 \leqslant n \leqslant z / \varepsilon} T^{-n}([0, \varepsilon])\right)=\nu(A(0,[z / \varepsilon], \varepsilon)),
$$

where $[t]=\max \{n \leqslant t, n \in N\}$. By this, for $z_{1}>z_{2}$, we have

$$
\begin{aligned}
\left|F_{\varepsilon}\left(z_{1} / \varepsilon\right)-F\left(z_{2} / \varepsilon\right)\right| & \leqslant \nu\left(A\left(\left[z_{2} / \varepsilon\right],\left[z_{1} / \varepsilon\right], \varepsilon\right)\right) \leqslant \sum_{n=\left[z_{2} / \varepsilon\right]}^{\left[z_{1} / \varepsilon\right]} \nu\left(T^{-n}([0, \varepsilon])\right) \\
& =\nu([0, \varepsilon])\left(\left[z_{1} / \varepsilon\right]-\left[z_{2} / \varepsilon\right]\right) \leqslant\left(z_{1}-z_{2}+\varepsilon\right) c,
\end{aligned}
$$

where $c$ is given by (1). Therefore, for any sequence $\varepsilon_{n}$ converging to zero the sequence of functions $F_{\varepsilon_{n}}\left(z / \varepsilon_{n}\right)$ is quasi-equicontinuous on $[0, \infty)$. Hence, from $F_{\varepsilon_{n}}\left(z / \varepsilon_{n}\right) \leqslant 1$, by the Arzela Theorem it follows that for any $\varepsilon_{n} \rightarrow 0$ and any $a>0$ there exists a subsequence $\varepsilon_{n_{j}}$ such that $F_{\varepsilon_{n_{j}}}\left(z / \varepsilon_{n_{j}}\right)$ is uniformly convergent on $[0, a]$. Now, let us assume that $F_{\varepsilon_{n}}\left(z / \varepsilon_{n}\right)$ is uniformly convergent on $[0, a]$ as $n \rightarrow \infty$ for some sequence $\varepsilon_{n} \rightarrow 0$ and some $a>0$. Denote by $G_{n}(z)(n=1,2, \ldots)$ the piecewise linear and continuous function with vertices at the points $\left(\left[z / \varepsilon_{n}\right] \varepsilon_{n}, F_{\varepsilon_{n}}\left(z / \varepsilon_{n}\right)\right)$. It is obvious that the function $G_{n}(z)$ is an approximation of the piecewise constant function $F_{\varepsilon_{n}}\left(z / \varepsilon_{n}\right)$ and, moreover,

$$
\left|G_{n}(z)-F_{\varepsilon_{n}}\left(z / \varepsilon_{n}\right)\right| \leqslant\left|F_{\varepsilon_{n}}\left(\left(z+\varepsilon_{n}\right) / \varepsilon_{n}\right)-F\left(z / \varepsilon_{n}\right)\right| \leqslant \nu\left(\left[0, \varepsilon_{n}\right]\right) \leqslant c \varepsilon_{n} .
$$

Consequently, the sequence $G_{n}(z)$ is uniformly convergent on $[0, a]$ to the same function as $F_{\varepsilon_{n}}\left(z / \varepsilon_{n}\right)$.

By definition of $G_{n}(z)$ the right and the left derivative of $G_{n}$ are given by formulas

$$
\begin{aligned}
G_{n+}^{\prime}(z) & =\frac{F_{\varepsilon_{n}}\left(\left(z+\varepsilon_{n}\right) / \varepsilon_{n}\right)-F_{\varepsilon_{n}}\left(z / \varepsilon_{n}\right)}{\varepsilon_{n}} \\
& =\frac{\nu\left(A\left(0,\left[z / \varepsilon_{n}\right]+1, \varepsilon_{n}\right)\right)-\nu\left(A\left(0,\left[z / \varepsilon_{n}\right], \varepsilon_{n}\right)\right)}{\varepsilon_{n}}
\end{aligned}
$$

whenever $z \in\left[k \varepsilon_{n},(k+1) \varepsilon_{n}\right) \cap[0, a], k=0,1, \ldots$, and

$$
\begin{aligned}
G_{n-}^{\prime}(z) & =\frac{F_{\varepsilon_{n}}\left(\left(z+\varepsilon_{n}\right) / \varepsilon_{n}\right)-F_{\varepsilon_{n}}\left(z / \varepsilon_{n}\right)}{\varepsilon_{n}} \\
& =\frac{\nu\left(A\left(0,\left[z / \varepsilon_{n}\right]+1, \varepsilon_{n}\right)\right)-\nu\left(A\left(0,\left[z / \varepsilon_{n}\right], \varepsilon_{n}\right)\right)}{\varepsilon_{n}}
\end{aligned}
$$

whenever $z \in\left(k \varepsilon_{n},(k+1) \varepsilon_{n}\right] \cap[0, a]$. Therefore, by Lemma 5 , for any $a \in(0, a]$ and for any sufficiently large $n$, we have

$$
\begin{aligned}
G_{n+}^{\prime}(z)= & \frac{\left[\nu\left(\left[0, \varepsilon_{n}\right]\right)-\nu\left(\varphi_{1}\left(\left[0, \varepsilon_{n}\right]\right)\right)\right]}{\varepsilon_{n}} \\
& \times\left[1-\nu\left(A\left(0,\left[z / \varepsilon_{n}\right]-u\left(\varepsilon_{n}\right)+1, \varepsilon_{n}\right)\right)\right] \\
& +R\left(\left[z / \varepsilon_{n}\right]-u\left(\varepsilon_{n}\right)+1,\left[z / \varepsilon_{n}\right], \varphi_{1}\left(\varepsilon_{n}\right)\right) \nu\left(\left[0, \varphi_{1}\left(\varepsilon_{n}\right)\right]\right) \varepsilon_{n}^{-1} \\
& -R\left(\left[z / \varepsilon_{n}\right]-u\left(\varepsilon_{n}\right)+1,\left[z / \varepsilon_{n}\right]+1, \varepsilon_{n}\right) \nu\left(\left[0, \varepsilon_{n}\right]\right) \varepsilon_{n}^{-1} .
\end{aligned}
$$


Since $g_{\nu}$ is continuous, we have

(21)

$$
\begin{aligned}
\lim _{n \rightarrow \infty} \frac{\nu\left(\left[0, \varepsilon_{n}\right]\right)-\nu\left(\varphi_{1}\left(\left[0, \varepsilon_{n}\right]\right)\right)}{\varepsilon_{n}} & =\lim _{n \rightarrow \infty} \frac{\nu\left(\left[0, \varepsilon_{n}\right]\right)}{\varepsilon_{n}}-\frac{\nu\left(\varphi_{1}\left(\left[0, \varepsilon_{n}\right]\right)\right) \varphi_{1}\left(\varepsilon_{n}\right)}{\varphi_{1}\left(\varepsilon_{n}\right) \varepsilon_{n}} \\
& =g_{\nu}(0)-g_{\nu}(0) \varphi_{1}^{\prime}(0) .
\end{aligned}
$$

Moreover, since $\varphi_{1}^{u\left(\varepsilon_{n}\right)}\left(a_{1}\right) \geqslant \varepsilon_{n}$,

$$
\begin{aligned}
\nu(A & \left.\left(0,\left[z / \varepsilon_{n}\right], \varepsilon_{n}\right)\right)-\nu\left(A\left(0,\left[z / \varepsilon_{n}\right]-u\left(\varepsilon_{n}\right)+1, \varepsilon_{n}\right)\right) \\
& \leqslant \nu\left(A\left(\left[z / \varepsilon_{n}\right]-u\left(\varepsilon_{n}\right),\left[z / \varepsilon_{n}\right], \varepsilon_{n}\right)\right) \leqslant u\left(\varepsilon_{n}\right) \nu\left(\left[0, \varepsilon_{n}\right]\right) \\
& \leqslant c u\left(\varepsilon_{n}\right) \mu\left(\left[0, \varepsilon_{n}\right]\right) \leqslant c u\left(\varepsilon_{n}\right) \mu\left(\left[0, \varphi_{1}^{u\left(\varepsilon_{n}\right)}\left(a_{1}\right)\right]\right) \\
& \leqslant c u\left(\varepsilon_{n}\right)\left(\max \varphi_{1}^{\prime}\right)^{u\left(\varepsilon_{n}\right)} \leqslant c u\left(\varepsilon_{n}\right) s_{0}^{-u\left(\varepsilon_{n}\right)}
\end{aligned}
$$

and, consequently,

(22)

$$
\begin{aligned}
\lim _{n \rightarrow \infty}\left(1-\nu\left(A\left(0,\left[z / \varepsilon_{n}\right]-u\left(\varepsilon_{n}\right)+1, \varepsilon_{n}\right)\right)\right) & =\lim _{n \rightarrow \infty}\left(1-\nu\left(A\left(0,\left[z / \varepsilon_{n}\right], \varepsilon_{n}\right)\right)\right) \\
& =1-\lim _{n \rightarrow \infty} F_{\varepsilon_{n}}\left(z / \varepsilon_{n}\right)=1-F(z),
\end{aligned}
$$

where $F(z)=\lim _{n \rightarrow \infty} F_{\varepsilon_{n}}\left(z / \varepsilon_{n}\right)$. From (20)-(22) and Lemma 4 it follows that, for $z \in(0, a]$, there exists $\lim _{n \rightarrow \infty} G_{n+}^{\prime}(z)$ and

$$
\lim _{n \rightarrow \infty} G_{n+}^{\prime}(z)=\sigma(1-F(z)) \text {. }
$$

Moreover, since $F(0)=\lim _{n \rightarrow \infty} F_{\varepsilon_{n}}\left(0 / \varepsilon_{n}\right)=\lim _{n \rightarrow \infty} \nu\left(\left[0, \varepsilon_{n}\right]\right)=0$ and

$$
\begin{aligned}
G_{n+}^{\prime}(0) & =\frac{\nu\left(\left[0, \varepsilon_{n}\right] \cup T^{-1}\left(\left[0, \varepsilon_{n}\right]\right)\right)-\nu\left(\left[0, \varepsilon_{n}\right]\right)}{\varepsilon_{n}} \\
& =\frac{\nu\left(\left[0, \varepsilon_{n}\right]\right)-\nu\left(\left[0, \varphi_{1}\left(\left[0, \varepsilon_{n}\right]\right)\right)\right.}{\varepsilon_{n}}
\end{aligned}
$$

by (21), there exists $\lim _{n \rightarrow \infty} G_{n+}^{\prime}(0)$ and

$$
\lim _{n \rightarrow \infty} G_{n+}^{\prime}(z)=\sigma(1-F(z))
$$

also at $z=0$. Similarly, we may show that, for $z \in(0, a]$, there exists $\lim G_{n-}^{\prime}(z)$ and

$$
\lim _{n \rightarrow \infty} G_{n-}^{\prime}(z)=\sigma(1-F(z)) \text {. }
$$

Consequently, by Lemma 6 , for $z \in[0, a]$, we have

$$
F^{\prime}(z)=\sigma(1-F(z)) \text {. }
$$

Therefore, since $F(0)=0$, for $z \in[0, a]$ the function $F(z)$ is given by the formula

$$
F(z)=1-e^{-\sigma z} \text {. }
$$

Hence, by Lemma 7, we obtain the thesis of the lemma. 
Proof of Theorem 1. For any nonnegative $f \in L^{1}$ such that $\|f\|=1$, we have

$$
\begin{aligned}
\mid F_{\varepsilon}(f, z / \varepsilon)-F_{\varepsilon} & \left.g_{\nu}, z / \varepsilon\right)|=| \mu_{f}(A(0,[z / \varepsilon], \varepsilon))-\nu(A(0,[z / \varepsilon], \varepsilon)) \mid \\
= & \left|\int_{A(0,[z / \varepsilon], \varepsilon)} f d \mu-\int_{A(0,[z / \varepsilon], \varepsilon)} g_{\nu} d \mu\right| \\
\leqslant & \left|\int_{A(0,[z / \varepsilon], \varepsilon)} f d \mu-\int_{A(q,[z / \varepsilon], \varepsilon)} f d \mu\right| \\
& +\left|\int_{A(q,[z / \varepsilon], \varepsilon)} f d \mu-\int_{A(q,[z / \varepsilon], \varepsilon)} g_{\nu} d \nu\right| \\
& +\left|\int_{A(q,[z / \varepsilon], \varepsilon)} g_{\nu} d \mu-\int_{A(0,[z / \varepsilon], \varepsilon)} g_{\nu} d \mu\right| \\
\leqslant & \int_{A(0, q, \varepsilon)} f d \mu+\left|\int_{A(0,[z / \varepsilon]-q, \varepsilon)}\left(P_{T}^{q} f-g_{\nu}\right) d \mu\right|+\int_{A(0, q, \varepsilon)} g_{\nu} d \mu .
\end{aligned}
$$

Since $P_{T}^{q} f$ is convergent in $L^{1}$-norm to $g_{\nu}$ (see [4]), from the last inequality with $q=[\ln [z / \varepsilon]]$ and from Lemma 8 we obtain the thesis of Theorem 1 .

4. Final remarks. Let $A_{\varepsilon} \subset[0,1]$ be an interval with $\mu\left(A_{\varepsilon}\right)=\varepsilon$. Put $\xi_{A_{\varepsilon}}(x)$ $=\min \left\{n: T^{n}(x) \in A_{\varepsilon}\right\}$ and $F_{\varepsilon}(f, z / \varepsilon)=\mu_{f}\left\{x: \xi_{A_{\varepsilon}}(x) \leqslant z / \varepsilon\right\}$, where $f \in L^{1}, f \geqslant 0$ and $\|f\|=1$. Then, under additional assumptions about the family of sets $A_{\varepsilon}$, we may prove the theorem similar to Theorem 1; that is, there exists $\sigma>0$ such that

$$
\lim _{\varepsilon \rightarrow 0} F_{\varepsilon}(f, z / \varepsilon)=1-e^{-\sigma z} .
$$

This is true, for example, if there exists $x_{0}$ such that $x_{0}$ is the left end point of any interval $A_{\varepsilon}$ and there exists $k$ such that $T^{k}\left(x_{0}\right)=x_{0}$.

We may state a similar theorem for expanding mappings $T: M \rightarrow M$ of a connected $C^{\infty}$-manifold into itself. The proof of such a theorem is similar to the proof of Theorem 1, but presents more technical difficulties.

\section{REFERENCES}

1. A. Lasota and J. A. Yorke, The law of exponential decay for expanding mappings, Rend. Sem. Mat. Univ. Padova 64 (1981), 141-157.

2. On the existence of invariant measures for piecewise monotonic transformations, Trans. Amer. Math. Soc. 186 (1973), 481-488.

3. A. Renyi, Representation for real numbers and their ergodic properties, Acta Math. Acad. Aci. Hungar. 8 (1952), 477-493.

4. A. Lasota, A fixed point theorem and its application in ergodic theory, Tôhoku Math. J. (2) 32 (1980), $567-575$

5. M. Jablonski, Z. S. Kowalski and J. Malczak, The rate of convergence of iterates of the Frobenius-Perron operator for Lasota-Yorke transformations, Univ. Jagello. Acta Math. (in press).

6. G. Pianigiani and J. A. Yorke, Expanding maps on sets which are almost invariant: decay and chaos, Trans. Amer. Math. Soc. 252 (1979), 351-366.

7. K. Krzyżewski and W. Szlenk, On invariant measures for expanding differentiable mappings, Studia Math. 33 (1969), 83-92.

8. M. Halfant, Analytic properties of Rényi's invariant density, Israel J. Math. (1977), 1-20.

9. A. Lasota, Ergodic problems in biology, Soc. Math. France Astérisque 50 (1977), 239-250.

10. J. A. Yorke and E. Yorke, Metastable chaos: The transition to sustained chaotic behavior in Lorenz model, J. Statist. Phys. 21 (1979), 263-277. 
11. K. Robbins, $A$ new approach to subcritical instability and turbulent transitions in a simple dynamo, Math. Proc. Cambridge Philos. Soc. 82 (1977), 309-325.

12. J. Kaplan and J. A. Yorke, Preturbulence: A regime observed in a fluid flow model of Lorenz, Comm. Math. Phys. 67 (1979), 93-103.

13. S. D. Poisson, Recherches sur la probabilité des jugements, Paris, 1837.

14. A. Avez, Propriétés ergodiques des endomorphismes dilatants des variétés compactes, C. R. Acad. Sci. Paris Sér. A-B 266 (1968), 610-612.

15. R. Bowen and D. Ruelle, The ergodic theory of Axiom A flow, Invent. Math. 29 (1975), 181-202.

16. E. Lorenz, Deterministic nonperiodic flow, J. Atmospheric Sci. 20 (1963), 130-141.

17. A. Lasota and M. C. Mackey, The extinction of slowly evolving dynamical systems, J. Math. Bio. 10 (1980), 333-345.

INSTITUTE OF INFORMATICS, JAgIELlONIAN UNIVERSITY, KRAKóW, POLAND 